ITP-SB-96-18

May, 1996

\title{
Some New Results on Complex-Temperature Singularities in Potts Models on the Square Lattice
}

\author{
Victor Matveev and Robert Shrock \\ Institute for Theoretical Physics \\ State University of New York \\ Stony Brook, N. Y. 11794-3840
}

\begin{abstract}
We report some new results on the complex-temperature (CT) singularities of $q$-state Potts models on the square lattice. We concentrate on the problematic region $\operatorname{Re}(a)<0$ (where $a=$ $\left.e^{K}\right)$ in which $\mathrm{CT}$ zeros of the partition function are sensitive to finite lattice artifacts. From analyses of low-temperature series expansions for $3 \leq q \leq 8$, we establish the existence, in this region, of complex-conjugate $\mathrm{CT}$ singularities at which the magnetization and susceptibility diverge. From calculations of zeros of the partition function, we obtain evidence consistent with the inference that these singularities occur at endpoints $a_{e}, a_{e}^{*}$ of arcs protruding into the (complex-temperature extension of the) FM phase. Exponents for these singularities are determined; e.g., for $q=3$, we find $\beta_{e}=-0.125(1)$, consistent with $\beta_{e}=-1 / 8$. By duality, these results also imply associated arcs extending to the (CT extension of the) symmetric PM phase. Analytic expressions are suggested for the positions of some of these singularities; e.g., for $q=5$, our finding is consistent with the exact value $a_{e}, a_{e}^{*}=2(-1 \mp i)$. Further discussions of complex-temperature phase diagrams are given.
\end{abstract}

*email: vmatveev@insti.physics.sunysb.edu

**email: shrock@insti.physics.sunysb.edu 


\section{Introduction and Model}

In this paper, we report some new results on complex-temperature singularities of the $q$-state Potts model [1, 2] on the square lattice. The Potts model has been of interest both as an example of a particular universality class for critical phenomena and as a model for physical phenomena such as the adsorption of certain gases on substrates [3]. However, in contrast to the 2D Ising model (equivalent to the $q=2$ case) the free energy of the Potts model for general $q$ has never been calculated in closed form, even for zero external field(s). Some exact results have been established for the model: from a duality relation, the critical point separating the disordered, $Z_{q}$-symmetric high-temperature phase from the low-temperature phase with spontaneously broken $Z_{q}$ symmetry and associated nonzero ferromagnetic (FM) long-range order is known [1]. The free energy and latent heat [4], and magnetization [5] have been calculated exactly by Baxter at this critical point, establishing that the model has a continuous, second-order transition for $q \leq 4$ and a first-order transition for $q \geq 5$. Baxter has also shown that although the $q=3$ model has no phase with antiferromagnetic (AFM) long-range order at any finite temperature, there is an AFM critical point at $T=0$ [5]. The values of the critical exponents (for the range of $q$ where the transition is continuous) have been determined [6]. Subsequently, further insight into the critical behavior was gained using the methods of conformal field theory [7]. A review of work up through 1982 was given in Ref. [8].

In general, if one knew the exact (zero-field) free energy, one would be able to determine the full phase diagram as a function of complex temperature. The idea of generalizing a variable on which the free energy depends from real physical values to complex values was pioneered by Yang and Lee [9]. These authors considered the generalization of the external magnetic field to complex values [9] and proved a celebrated theorem that the complexfield zeros of the Ising model partition function lie on the unit circle in the $\mu$ plane, where $\mu=e^{-2 \beta H}$, pinching the real axis as the temperature $T$ decreases through the critical point $T_{c}$. Complex-temperature $(\mathrm{CT})$ singularities of Ising models, first considered in Ref. [10], were investigated both by means of CT zeros of the partition function [11-13 and via their effects on low-temperature series expansions [14]. As well as being of historical interest, these are relevant here because of the equivalence of the (spin 1/2) Ising model and $q=2$ Potts model. There is continuing interest in such complexifications because of the deeper insight which they give one into the properties of statistical mechanical models (for the Ising model, see, e.g., Refs. [15]-26]). From general arguments and comparisons with exact solutions for 2D Ising models with isotropic couplings, one knows that in the thermodynamic limit, CT zeros merge together to form curves (including possible line segments) across which the free 
energy is non-analytic. These curves form the complex-temperature phase boundary (CTPB) $\mathcal{B}$ of the model. One can define notions of complex-temperature extensions (CTE's) of the physical paramagnetic (PM), ferromagnetic (FM) and (if it occurs) antiferromagnetic (AFM) phases. In certain cases there are other (labelled "O") complex-temperature phases which do not have any overlap with any physical phase. These various CT phases are separated by boundaries comprising $\mathcal{B}$. The locus of points making up $\mathcal{B}$ may also contain part(s) consisting of curves (arcs) or line segments which protrude into and terminate in, certain phases.

There have been several calculations of complex-temperature zeros of the partition function for the Potts model on the square lattice [27]-32]. Since the early calculations for $q=3,4$, it has been recognized that the zeros show one clear feature: if one uses dualitypreserving boundary conditions, then in the $R e(a)>0$ region (where $a=e^{K}$; see below for notation), these zeros lie on a portion of the unit circle $|x|=1$, where $x=(a-1) / \sqrt{q}$ [28-31. In passing, we note that in the $q \rightarrow \infty$ limit it has been shown (assuming that the $q \rightarrow \infty$ limit and the thermodynamic limit commute) that the CT zeros lie on the unit circle $|x|=1$ 32, 33]. However, for a given (finite) $q$, the situation in the $\operatorname{Re}(a)<0$ region has proved to be much more difficult to elucidate. The zeros exhibit considerable scatter and, as we shall demonstrate, significant sensitivity to the boundary conditions used for the finite lattice calculations, even if one requires these to preserve duality. These facts have rendered it problematic to try to make inferences from calculations of zeros on finite lattices about the complex-temperature phase boundary $\mathcal{B}$ in the thermodynamic limit. In one early work [27] it was conjectured that in the thermodynamic limit the zeros lie on the two circles $|a-1|=\sqrt{q}$ and $|a+1|=\sqrt{4-q}$ for $q=3$ and $q=4$ (where the second circle degenerates to a point), but shortly thereafter, from a calculation of zeros for the $q=3$ model on larger lattices, it was concluded that this conjecture was false [28 and the zero distribution did not suggest the existence of any simple algebraic expression which would describe this distribution. The same conclusion was reached from a calculation of the zeros for the $q=4$ model 29].

We have been able to make progress in the problematic $\operatorname{Re}(a)<0$ region by employing a powerful method not hitherto used for this purpose, viz., to combine analyses of lowtemperature series with calculations of CT zeros of the partition function. We report our results here. In our series work, we have taken advantage of the recent calculations of quite long low-temperature series for the free energy, magnetization, and susceptibility of the square-lattice Potts model for $q=3$ up to $q=10$ by Briggs, Enting, and Guttmann [35], extending earlier calculations (e.g., Ref. [36]; for $q=3$ and $q=8$, see also Ref. [37].) The organization of this paper is as follows. In section 2 we define the model and our notation 
and mention some of the general exact results which are known. In sections 3 and 4 we present our new results for the $q=3$ and $q=4$ Potts model. In section 5 we mention some similar results for $q \geq 5$ Potts models. Concluding remarks are given in section 6 .

\section{Definition of Model and Exact Results}

The (isotropic, nearest-neighbor) $q$-state Potts model on a lattice $\Lambda$ is defined, at a temperature $T$, by the partition function

$$
Z=\sum_{\left\{\sigma_{n}\right\}} e^{-\beta \mathcal{H}}
$$

with the Hamiltonian

$$
\mathcal{H}=-J \sum_{\left\langle n n^{\prime}\right\rangle} \delta_{\sigma_{n} \sigma_{n^{\prime}}}-H \sum_{n} \delta_{0} \sigma_{n}
$$

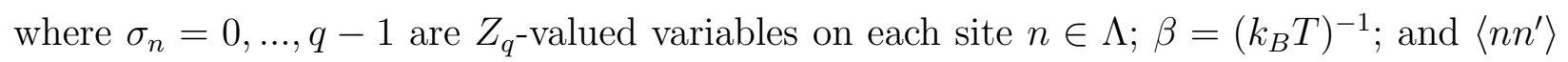
denotes pairs of nearest-neighbor sites. We use the notation $K=\beta J, h=\beta H$,

$$
a=z^{-1}=e^{K}
$$

and

$$
x=\frac{e^{K}-1}{\sqrt{q}}
$$

and denote the (reduced) free energy per site as $f=-\beta F=\lim _{N_{s} \rightarrow \infty} N_{s}^{-1} \ln Z$, where $N_{s}$ denotes the number of sites in the lattice. Here we consider the square (sq) lattice. There are actually $q$ types of external fields which one may define, favoring the respective values $\sigma_{n}=0 .,,, q-1$; it suffices for our purposes to include only one. The order parameter is defined to be

$$
m=\frac{q M-1}{q-1}
$$

where $M=\langle\sigma\rangle=\lim _{h \rightarrow 0} \partial f / \partial h$. With this definition, $m=0$ in the $Z_{q}$-symmetric, disordered phase, and $m=1$ in the limit of saturated ferromagnetic (FM) long-range order. We shall refer to $m$ as the magnetization. Finally, the (reduced, initial) susceptibility is denoted as $\bar{\chi}=\beta^{-1} \chi=\partial m /\left.\partial h\right|_{h=0}$. We consider the zero-field model, $H=0$, unless otherwise stated.

The general $q$-state Potts model on the square lattice obeys the duality relation [1]

$$
(a-1)\left(a_{d}-1\right)=q
$$

where $a_{d} \equiv \mathcal{D}(a)$ is the image under the duality map $\mathcal{D}$ of $a$;

$$
\mathcal{D}(a)=1+\frac{q}{a-1}
$$


with $\mathcal{D}^{2}=1$ as usual. In terms of the variable $x$, this duality relation takes the simple form

$$
x_{d}=\frac{1}{x}
$$

The critical point at which a phase transition occurs between the high-temperature, symmetric phase and the low-temperature, FM phase, is given by the self-dual point $x_{c}=1$, i.e., $a_{c}=1+\sqrt{q}$. One may observe that eq. (6) also has a second self-dual solution at a complex-temperature point,

$$
a=1-\sqrt{q} \equiv a_{\ell}
$$

Exact expressions for the free and internal energy, latent heat, and magnetization have been given by Baxter [由, 5] on the critical self-dual curve

$$
(a-1)^{2}=q
$$

Since the latent heat is zero at $a_{c}$ for $q \leq 4$ [ []], the corresponding transition between the symmetric and FM phases is continuous. The curve (10) also includes the complextemperature singular point at $a_{\ell}$ in eq. (9). We note that by the same reasoning, the phase boundary associated with the complex-temperature point $a_{\ell}$ is also continuous. Exact results have also been given by Baxter for the critical manifold [5, 38]

$$
(a+1)^{2}=4-q
$$

For $q=3$, eq. (11) has two solutions: the AFM critical point at $a=0$, i.e. $T=0$ with $J<0$, and a complex-temperature point,

$$
a=-2 \equiv a_{s}
$$

We observe that these two points are mapped into each other under the duality mapping $\mathcal{D}$ :

$$
\mathcal{D}(a=0)=-2
$$

For $q=4$, eq. (11) has only the solution $a=-1$, implying that for $q \geq 4$ the model has no AFM long-range order even at $T=0$.

Consider an $L_{x} \times L_{y}$ planar square lattice $G$ with $N_{s} \equiv N_{0}$ sites (0-cells), $N_{b} \equiv N_{1}$ bonds (1-cells) and $N_{p} \equiv N_{2}$ plaqettes (2-cells). We shall discuss boundary conditions in section 3.5 below. The dual of $G$, which we denote $G_{d}=\mathcal{D}(G)$, is defined by associating uniquely a $(2-p)$-cell of $G_{d}$ with each $p$-cell of $G$, so that $\left(N_{0}\right)_{G}=\left(N_{2}\right)_{G_{d}},\left(N_{1}\right)_{G}=\left(N_{1}\right)_{G_{d}}$, and $\left(N_{2}\right)_{G}=\left(N_{0}\right)_{G_{d}}$. Since the planar graph has no handles, $N_{h}=0$ and hence Euler characteristic $\chi_{E}=2\left(1-N_{h}\right)=2$, it follows from the Euler relation $\sum_{j=0}^{2}(-1)^{j} N_{j}=\chi_{E}$ 
that $N_{0}-N_{1}+N_{2}=2$. The duality relation connecting the partition function $Z_{G}$ on $G$ with that on $G_{d}$ is $[8]$

$$
Z_{G}(x)=x^{N_{1}} q^{N_{0}-1-(1 / 2) N_{1}} Z_{G_{d}}\left(x_{d}\right)
$$

It follows from (14) that in the thermodynamic limit, the singularities of that the free energy at a point $a$ and its dual image $a_{d}$ are the same. In particular,

$$
f_{\text {sing }}(q=3 ; a=-2)=f_{\text {sing }}(q=3 ; a=0)
$$

In discussing the complex-temperature phase diagram, it is convenient to use the Boltzmann weight $z$ and its inverse $a$, and the related variable, $x$. For $q=3$, the exact results discussed above show that the phase structure for physical temperature (i.e. $0 \leq a \leq \infty$ ) consists of (i) the disordered, $Z_{3}$-symmetric PM phase for $0<a \leq 1+\sqrt{3}$; (ii) the FM phase for $1+\sqrt{3}<a \leq \infty$; and (iii) an AFM critical point at $a=0$. For $q \geq 4$, the physical phase structure consists only of the PM phase for $0 \leq a \leq 1+\sqrt{q}$ and the FM phase for $1+\sqrt{q}<a \leq \infty$. One defines the complex-temperature extensions (CTE's) of the PM and FM phases by analytically continuing away from the respective segments of the positive real $a$ axis. Two rigorous properties are the following. First, because the model has a high-temperature series expansion with finite radius of convergence, it follows that the CTE of the PM phase occupies a finite neighborhood surrounding the point $a=1$. Second, it is easy to show that for sufficiently large $|a|$, one is necessarily in the (CTE of the) FM phase. To see this, let $a=\rho_{a} e^{i \theta_{a}}$; then

$$
K=\ln a=\ln \rho_{a}+i\left(\theta_{a}+2 \pi n\right)
$$

where $n$ denotes the Riemann sheet of the logarithm and may be taken equal to zero here. It is clear that for sufficiently large $|a|=\rho_{a}$, the angle $\theta_{a}$ makes a negligible contribution to $K$, so that (given that $d=2$ is above the lower critical dimensionality for the FM transition), the system will be in the FM phase. This fact can be seen equivalently as a consequence of the fact that the model has a low-temperature expansion with a finite radius of convergence, so that there is a finite neighborhood of the origin in the complex $z$ plane where it is in the (CTE of the) FM phase. Henceforth, we shall generally refer to the complex-temperature extension of the FM phase simply as the FM phase and similarly with the PM phase.

\section{$3 q=3$ Potts Model}

We shall discuss our methods in detail for the $q=3$ square-lattice Potts model and then proceed to the higher $q$ cases. We begin with analyses of the low-temperature series expansions. The series for the partition function, magnetization, and susceptibility have been 
calculated to order $z^{47}$ 35. As in our previous studies of complex-temperature singularities of various spin models, we have used both dlog Padé and differential approximants to analyze the series. We fit the specific heat $C$, the magnetization $m$, and the (reduced) susceptibility $\bar{\chi}$ to the leading singular forms applicable near a generic singular point $z_{\text {sing }}$ :

$$
\begin{aligned}
& C \sim\left(1-z / z_{\text {sing }}\right)^{-\alpha_{\text {sing }}^{\prime}} \\
& m \sim\left(1-z / z_{\text {sing }}\right)^{\beta_{\text {sing }}}
\end{aligned}
$$

and

$$
\bar{\chi} \sim\left(1-z / z_{\text {sing }}\right)^{-\gamma_{\text {sing }}^{\prime}}
$$

As usual, the primes indicate that we are approaching this singularity from within the interior of the FM phase. We find convincing evidence from our series analyses for singularities at two complex-conjugate points, which we denote $z_{e}$ and $z_{e}^{*}$, at which the magnetization, susceptibility, and specific heat are divergent.

\subsection{Magnetization}

In Table 1 we present some diagonal and near-diagonal dlog Padé results for this singularity. (We have, of course, also calculated approximants farther from the diagonal.) Our analysis of the series for the order parameter indicates complex-conjugate singularities at approximately $z_{e}, z_{e}^{*}=-0.34 \pm 0.29 i$ (the location will be discussed further below) where $m$ diverges with the exponent

$$
\beta_{e}=-0.125(1)
$$

A plausible inference is that the exact value of this exponent is

$$
\beta_{e}=-\frac{1}{8}
$$

We comment that although such a divergence in the order parameter is forbidden in usual physical phase transitions, it can and does occur at complex-temperature singularities. Indeed, in our previous work we have noted several instances where the magnetization diverges at CT singularities. For example, exact results show that $M$ diverges (like $(1+3 u)^{-1 / 8}$ ) at the CT point $u=-1 / 3$ in the (zero-field) spin $1 / 2$ Ising model on the triangular lattice and at $u=u_{e}=-(3-2 \sqrt{2})$ in the Ising model with $\beta H=i \pi / 2$ on the square lattice (like $\left.\left(1-u / u_{e}\right)^{-1 / 8}\right)[22$.

The appearance of the exponent (20) at this CT singularity in the square-lattice Potts model is intriguing, since $-1 / 8$ is not a simple (negative) multiple of any of the physical magnetic exponents in the model. This contrasts with the above-mentioned examples from 


\begin{tabular}{|l|l|l|}
\hline \hline$[N / D]$ & $z_{e}$ & $\beta_{e}$ \\
\hline$[13 / 12]$ & $-0.340504+0.287457 i$ & -0.1258 \\
\hline$[13 / 13]$ & $-0.340420+0.287491 i$ & -0.1256 \\
\hline$[14 / 13]$ & $-0.340382+0.287392 i$ & -0.1250 \\
\hline$[13 / 14]$ & $-0.340358+0.287473 i$ & -0.1252 \\
\hline$[14 / 14]$ & $-0.340441+0.287408 i$ & -0.1254 \\
\hline$[15 / 14]$ & $-0.340428+0.287382 i$ & -0.1252 \\
\hline$[14 / 15]$ & $-0.340427+0.287365 i$ & -0.1251 \\
\hline$[15 / 15]$ & $-0.340433+0.287339 i$ & -0.1251 \\
\hline$[16 / 15]$ & $-0.340436+0.287386 i$ & -0.1253 \\
\hline$[15 / 16]$ & $-0.340429+0.287368 i$ & -0.1252 \\
\hline$[16 / 16]$ & $-0.340512+0.287332 i$ & -0.1256 \\
\hline$[17 / 16]$ & $-0.340405+0.287364 i$ & -0.1250 \\
\hline$[16 / 17]$ & $-0.340412+0.287339 i$ & -0.1250 \\
\hline$[17 / 17]$ & $-0.340406+0.287354 i$ & -0.1250 \\
\hline$[18 / 17]$ & $-0.340416+0.287357 i$ & -0.1251 \\
\hline$[17 / 18]$ & $-0.340410+0.287350 i$ & -0.1250 \\
\hline$[18 / 18]$ & $-0.340406+0.287356 i$ & -0.1250 \\
\hline$[19 / 18]$ & $-0.340407+0.287363 i$ & -0.1250 \\
\hline$[18 / 19]$ & $-0.340376+0.287413 i$ & -0.1251 \\
\hline$[19 / 19]$ & $-0.340312+0.287325 i$ & -0.1244 \\
\hline$[20 / 19]$ & $-0.340260+0.287344 i$ & -0.1241 \\
\hline$[19 / 20]$ & $-0.340273+0.287344 i$ & -0.1242 \\
\hline$[20 / 20]$ & $-0.340305+0.287229 i$ & -0.1241 \\
\hline$[21 / 20]$ & $-0.340285+0.287321 i$ & -0.1242 \\
\hline$[20 / 21]$ & $-0.340292+0.287319 i$ & -0.1243 \\
\hline$[21 / 21]$ & $-0.340326+0.287269 i$ & -0.1243 \\
\hline$[22 / 21]$ & $-0.340711+0.286857 i$ & -0.1246 \\
\hline$[22 / 22]$ & $-0.340855+0.286878 i$ & -0.1247 \\
\hline$[23 / 22]$ & $-0.340688+0.286888 i$ & -0.1246 \\
\hline$[22 / 23]$ & $-0.340982+0.287191 i$ & -0.1260 \\
\hline$[23 / 23]$ & $-0.340806+0.287311 i$ & -0.1262 \\
\hline \hline & & \\
\hline
\end{tabular}

Table 1: Values of $z_{e}$ and $\beta_{e}$ from dlog Padé approximants to low-temperature series for $m$ for $q=3$. 
the 2D Ising model, where, as is clear from the exact solution, the divergent magnetic exponents at $u=-1 / 3$ on the triangular lattice and at $u=u_{e}$ on the square lattice for $h=i \pi / 2$ are precisely minus the common value of $\beta=1 / 8$ at the physical PM-FM critical point. Specifically, for the PM-FM transition in the $2 \mathrm{D} q=3$ Potts model, the thermal and magnetic exponents are $y_{t}=1 / \nu=1 / \nu^{\prime}=6 / 5$ and $y_{h}=28 / 15$, whence $\alpha=\alpha^{\prime}=$ $2-d / y_{t}=1 / 3, \delta=\left(d / y_{h}-1\right)^{-1}=14, \beta=1 / 9, \gamma=\gamma^{\prime}=13 / 9$, and $\eta=4 / 15$ [6]. One recalls that the exponent $1 / 8$ does occur in the set of the conformal weights for the $m=5$ conformal field theory relevant to the $2 \mathrm{D} q=3$ Potts model, viz., $h_{1,2}=h_{4,4}=1 / 8$, where $h_{p, q}=\left[((m+1) p-m q)^{2}-1\right] /[4 m(m+1)]$ for $p=1, \ldots, m-1, q=1, \ldots, p$, and where the central charge is given by $c=1-6 /[m(m+1)]$ and has the value $c=4 / 5$ for this case [7]. However, the relation of this to the appearance of the exponent $-1 / 8$ at the complextemperature singularities $z_{e}, z_{e}^{*}$ is obscure, for several reasons. First, as we discussed in Ref. [19], there are violations of basic scaling relations at complex-temperature singularities, so that it is not clear how to apply conformal field theory to such singularities (since CFT implies, among other things, such scaling relations). Secondly, since the Hamiltonian is not real at complex-temperature singularities, it is not obvious why the unitary rational conformal series is relevant to such singularities.

\section{$3.2 \quad$ Susceptibility}

In Table 2 we present our corresponding results from the dlog Padé analysis of the lowtemperature series for the susceptibility. We have also carried out a similar study with (first-order, unbiased) differential approximants, which yields the same value, to within the uncertainty. We determine the value of the specific heat exponent at the singularities $z_{e}, z_{e}^{*}$ to be

$$
\gamma_{e}^{\prime}=1.14(6)
$$

where the uncertainty represents a theoretical estimate from the scatter of values among different Padé and differential approximants.

\subsection{Specific Heat}

To study the complex-temperature singularities in the specific heat, we have again carried out analyses with both Padé and differential approximants. As an illustration, we show in Table 3 our results from the latter. Our notation is the same as in our earlier works, e.g. Ref. [19]; $\left[L / M_{0} ; M_{1}\right]$ is the differential approximant to the generic function $\phi(z)$ obtained as the solution to the ordinary differential equation $Q_{0}(z) \phi(z)+Q_{1}(z)(z d / d z) \phi(z)=R(z)$, 


\begin{tabular}{|c|c|c|}
\hline \hline$[N / D]$ & $z_{e}$ & $\gamma_{e}^{\prime}$ \\
\hline$[15 / 15]$ & $-0.337324+0.290677 i$ & 1.123 \\
\hline$[17 / 15]$ & $-0.339008+0.289393 i$ & 1.190 \\
\hline$[15 / 16]$ & $-0.339062+0.288573 i$ & 1.173 \\
\hline$[16 / 16]$ & $-0.338269+0.289319 i$ & 1.149 \\
\hline$[17 / 16]$ & $-0.338281+0.289318 i$ & 1.150 \\
\hline$[18 / 16]$ & $-0.338121+0.289177 i$ & 1.138 \\
\hline$[15 / 17]$ & $-0.337814+0.289264 i$ & 1.125 \\
\hline$[16 / 17]$ & $-0.338280+0.289318 i$ & 1.150 \\
\hline$[17 / 17]$ & $-0.338270+0.289319 i$ & 1.149 \\
\hline$[18 / 17]$ & $-0.338227+0.289314 i$ & 1.147 \\
\hline$[19 / 17]$ & $-0.338392+0.289613 i$ & 1.163 \\
\hline$[16 / 18]$ & $-0.338168+0.289263 i$ & 1.143 \\
\hline$[17 / 18]$ & $-0.338226+0.289312 i$ & 1.147 \\
\hline$[18 / 18]$ & $-0.338259+0.289325 i$ & 1.149 \\
\hline$[19 / 18]$ & $-0.338336+0.289377 i$ & 1.154 \\
\hline$[20 / 18]$ & $-0.338338+0.289242 i$ & 1.151 \\
\hline$[17 / 19]$ & $-0.338077+0.289273 i$ & 1.138 \\
\hline$[18 / 19]$ & $-0.338332+0.289360 i$ & 1.153 \\
\hline$[19 / 19]$ & $-0.338311+0.289333 i$ & 1.152 \\
\hline$[20 / 19]$ & $-0.338355+0.289392 i$ & 1.155 \\
\hline$[21 / 19]$ & $-0.338537+0.289589 i$ & 1.170 \\
\hline$[18 / 20]$ & $-0.338310+0.289320 i$ & 1.151 \\
\hline$[19 / 20]$ & $-0.338341+0.289364 i$ & 1.154 \\
\hline$[20 / 20]$ & $-0.338168+0.289333 i$ & 1.145 \\
\hline$[21 / 20]$ & $-0.337704+0.289629 i$ & 1.126 \\
\hline$[22 / 20]$ & $-0.337349+0.290843 i$ & 1.111 \\
\hline$[19 / 21]$ & $-0.338488+0.291209 i$ & 1.196 \\
\hline$[20 / 21]$ & $-0.337205+0.289884 i$ & 1.102 \\
\hline$[21 / 21]$ & $-0.340368+0.286150 i$ & 1.080 \\
\hline$[20 / 22]$ & $-0.336698+0.291093 i$ & 1.064 \\
\hline \hline & & \\
\hline
\end{tabular}

Table 2: Values of $z_{e}$ and $\gamma_{e}^{\prime}$ from dlog Padé approximants to low-temperature series for $\bar{\chi}$ for $q=3$. 
where $Q_{0}, Q_{1}$, and $R$ are polynomials of order $M_{0}, M_{1}$, and $L$. A review of the methods is given in Ref. 40. We determine the specific heat exponent to be

$$
\alpha_{e}^{\prime}=1.0(1)
$$

As can be seen from Tables 1-3, the magnetization, susceptibility, and specific heat series give consistent values for the location of the singularities $z_{e}, z_{e}^{*}$. Combining these, we infer that

$$
z_{e}, z_{e}^{*}=-0.339(2) \pm 0.289(2) i
$$

For our comparison with the plots of zeros of the partition function, it will be convenient to re-express this in terms of the $a$ and $x$ variables. We list the results in Table 4 (together with positions of the corresponding singularity for higher values of $q$, to be discussed later).

In passing, we observe that although we have found violations of scaling relations such as $\alpha+2 \beta+\gamma=2$ and $\alpha^{\prime}+2 \beta+\gamma^{\prime}=2$ in our previous work at various complex-temperature singularities (e.g., Refs. [19, 24]), in the present case, we obtain $\alpha_{e}^{\prime}+2 \beta_{e}+\gamma_{e}^{\prime}=1.9 \pm 0.1$ so that, to within the uncertainties, this exponent relation is satisfied.

\subsection{Singularities at Dual Images of $a_{e}, a_{e}^{*}$}

A rigorous consequence of the duality of the model is that the free energy also is singular, with the same singularity, at the points which are the dual images of the $a_{e}$ and $a_{e}^{*}$, namely, for the central values, $\mathcal{D}\left(a_{e}\right), \mathcal{D}\left(a_{e}^{*}\right)=0.141 \pm 0.462 i$ or equivalently, $\mathcal{D}\left(x_{e}\right), \mathcal{D}\left(x_{e}^{*}\right)=-0.496 \pm 0.267$. These points lie in the (CTE)PM phase. Note that since $\left|x_{c}\right|=1$ while $\left|x_{e}\right|=0.56$, the singularities at $x_{e}, x_{e}^{*}$ lie closer to the origin in the $x$ plane than the physical critical point.

\subsection{Connection of $a_{e}, a_{e}^{*}$ Singularities with CT Phase Boundary $\mathcal{B}$}

We would also like to relate these complex-conjugate CT singularities at $z_{e}, z_{e}^{*}$, or equivalently, in the complex $a$ plane, at $a_{e}, a_{e}^{*}$, to the complex-temperature phase boundary $\mathcal{B}$. From our previous studies on CT singularities [20, 22, 24], we formulated a conjecture that whenever an arc or line segment of the phase boundary protrudes into, and ends in, the FM phase, there is a divergence in $M$ at the endpoint of this arc. We proved that this divergence in $M$ implies also a divergence in $\chi$ at the same endpoint [24]. Besides the exact results alluded to above which exhibit this behavior, our calculations of partition function zeros for the 2D higher-spin square-lattice Ising model [23], in conjunction with the series analyses of Jensen, Guttmann, and Enting [26], are consistent with the conjecture. A natural extension of the conjecture is that the divergences which we have found in $m$ at these CT points in the 


\begin{tabular}{|c|c|c|}
\hline \hline$\left[L / M_{0} ; M_{1}\right]$ & $z_{e}$ & $\alpha_{e}^{\prime}$ \\
\hline \hline$[8 / 16 ; 14]$ & $-0.3407788+0.2885827 i$ & 1.072 \\
\hline$[8 / 16 ; 15]$ & $-0.3407563+0.2878427 i$ & 1.052 \\
\hline$[8 / 16 ; 16]$ & $-0.3395542+0.2877949 i$ & 0.997 \\
\hline$[8 / 16 ; 17]$ & $-0.3399969+0.2874121 i$ & 0.986 \\
\hline$[8 / 17 ; 15]$ & $-0.3393125+0.2891874 i$ & 0.960 \\
\hline$[8 / 17 ; 16]$ & $-0.3391475+0.2886514 i$ & 0.967 \\
\hline$[10 / 15 ; 13]$ & $-0.3405761+0.2887910 i$ & 1.049 \\
\hline$[10 / 15 ; 14]$ & $-0.3405514+0.2889317 i$ & 1.044 \\
\hline$[10 / 15 ; 15]$ & $-0.3400580+0.2896685 i$ & 0.987 \\
\hline$[10 / 15 ; 16]$ & $-0.3392558+0.2894922 i$ & 0.935 \\
\hline$[10 / 16 ; 14]$ & $-0.3396813+0.2885613 i$ & 0.989 \\
\hline$[10 / 16 ; 15]$ & $-0.3397607+0.2885435 i$ & 0.994 \\
\hline$[12 / 14 ; 12]$ & $-0.3401737+0.2893914 i$ & 1.001 \\
\hline$[12 / 14 ; 13]$ & $-0.3405823+0.2891851 i$ & 1.049 \\
\hline$[12 / 14 ; 14]$ & $-0.3406363+0.2897800 i$ & 1.059 \\
\hline$[12 / 14 ; 15]$ & $-0.3395286+0.2896329 i$ & 0.934 \\
\hline$[12 / 15 ; 13]$ & $-0.3407441+0.2896263 i$ & 1.071 \\
\hline$[12 / 15 ; 14]$ & $-0.3404562+0.2891536 i$ & 1.039 \\
\hline$[14 / 13 ; 11]$ & $-0.3399260+0.2888016 i$ & 0.989 \\
\hline$[14 / 13 ; 12]$ & $-0.3399969+0.2885408 i$ & 1.001 \\
\hline$[14 / 13 ; 13]$ & $-0.3395575+0.2895497 i$ & 0.919 \\
\hline$[14 / 13 ; 14]$ & $-0.3395242+0.2893949 i$ & 0.915 \\
\hline$[14 / 14 ; 12]$ & $-0.3405031+0.2889864 i$ & 1.030 \\
\hline$[14 / 14 ; 13]$ & $-0.3395982+0.2892116 i$ & 0.942 \\
\hline$[16 / 12 ; 10]$ & $-0.3394602+0.2889824 i$ & 0.955 \\
\hline$[16 / 12 ; 11]$ & $-0.3391883+0.2891054 i$ & 0.929 \\
\hline$[16 / 12 ; 12]$ & $-0.3394838+0.2892739 i$ & 0.939 \\
\hline$[16 / 12 ; 13]$ & $-0.3393726+0.2892381 i$ & 0.934 \\
\hline$[16 / 13 ; 11]$ & $-0.3394488+0.2892846 i$ & 0.937 \\
\hline$[16 / 13 ; 12]$ & $-0.3394364+0.2893045 i$ & 0.933 \\
\hline$[18 / 11 ; 9]$ & $-0.3404461+0.2882994 i$ & 1.032 \\
\hline$[18 / 11 ; 10]$ & $-0.3398013+0.2888885 i$ & 0.979 \\
\hline$[18 / 11 ; 11]$ & $-0.3395252+0.2888272 i$ & 0.962 \\
\hline$[18 / 11 ; 12]$ & $-0.3396986+0.2891254 i$ & 0.955 \\
\hline$[18 / 12 ; 10]$ & $-0.3394656+0.2892506 i$ & 0.939 \\
\hline$[18 / 12 ; 11]$ & $-0.3394441+0.2891136 i$ & 0.945 \\
\hline \hline & & \\
\hline
\end{tabular}

Table 3: Values of $z_{e}$ and $\alpha_{e}^{\prime}$ from differential approximants to low-temperature series for (reduced) specific heat, $C /\left(k_{B} K^{2}\right)$, for $q=3 ! 1$ 


\begin{tabular}{|c|c|c|c|}
\hline \hline$q$ & $z_{e}, z_{e}^{*}$ & $a_{e}, a_{e}^{*}$ & $x_{e}, x_{e}^{*}$ \\
\hline \hline 3 & $-0.339(2) \pm 0.289(2) i$ & $-1.71(1) \mp 1.46(1) i$ & $-1.56(1) \mp 0.841(3) i$ \\
\hline 4 & $-0.288(2) \pm 0.270(2) i$ & $-1.85(1) \mp 1.73(1)$ & $-1.42(1) \mp 0.866(4) i$ \\
\hline 5 & $-0.251(2) \pm 0.251(2) i$ & $-1.99(1) \mp 1.99(1) i$ & $-1.34(1) \mp 0.891(4) i$ \\
\hline \hline
\end{tabular}

Table 4: Values of $z_{e}$ and, correspondingly, $a_{e}$ and $x_{e}$ from analyses of low-temperature series for magnetization, susceptibility, and specific heat. See text for further details.

square-lattice $q$-state Potts model indicate that these points are endpoints of arcs of points where $f$ is non-analytic, i.e. arcs on the CT phase boundary $\mathcal{B}$.

In order to test this conjecture here, we have carried out new calculations of complextemperature zeros of the partition function for several different finite lattices, using transfer matrix methods, as in our earlier study of partition function zeros for the 2D higher-spin Ising model [23]. At appropriate points we shall make comparison with previous computations of zeros for the square-lattice Potts model [28]-[31]. It is desirable to, and we shall, restrict ourselves to lattices with duality-preserving boundary conditions (DBC's) [8, 30, 31]. These guarantee that $\mathcal{D}(G)=G$ i.e. the dual of a (finite) lattice $G$ is (isomorphic to) the original lattice. To discuss these, we recall eq. (14) and the associated definitions. Note that 2D periodic, i.e. toroidal, boundary conditions do not preserve duality for a finite lattice. The boundary conditions are not uniquely specified by the condition that they preserve duality. One type, which we label as type 1, was discussed in Ref. [31] (see their Fig. 1). We shall need a straightforward generalization of it to the case of an $L_{x} \times L_{y}$ lattice with $L_{x} \neq L_{y}$, and we describe this as follows. Let the lattice be oriented with the $x$ and $y$ directions being horizontal and vertical, respectively. Let all of the sites on the upper and right-hand edges, including the corners, connect along directions outward from the lattice to a common site adjoined to this lattice (so that the upper right corner connects to this adjoined point via bonds in both the $x$ and the $y$ directions), while all of the sites on the lower and left-hand edges, excluding the previously mentioned corners, have free boundary conditions. For the dual lattice, the special adjoined point may be taken to lie to the lower left of the lattice. In Ref. [31] it was noted that from (unpublished) calculations of zeros with other DBC's, they obtained agreement with their conclusions from type 1 DBC's that for $\operatorname{Re}(x)>0$ the zeros lie on the circle $|x|=1$ [31]. We have also used a set of DBC's different from type 1, which we denote as type 2 [34]. For these, let the $L_{x} \times L_{y}$ lattice have periodic boundary 
conditions (PBC's) in the $x$ direction, so that the lattice may be pictured as a cylinder with its axis oriented vertically. Now connect all of the sites on the upper edge of the cylinder to a special point adjoined to the lattice, and let all of the sites on the lower edge of the cylinder have free boundary conditions. The dual lattice is constructed in the usual way, assigning sites to each 2-cell of the original graph, and adjoining the special point below the cylinder with the stipulation that the points on the upper edge of the dual lattice have free boundary conditions and the points on the lower edge connect to the adjoined point. For both type 1 and type 2 DBC's, $N_{0}=L_{x} L_{y}+1=N_{2}$ and $N_{1}=2 L_{x} L_{y}$ so that the prefactor $q^{N_{0}-1-(1 / 2) N_{1}}=1$ in eq. (14). Note also that both type 1 and type 2 DBC's force some sites to have coordination number 3 rather than 4, and the adjoined point has coordination number $L_{x}+L_{y}$ for type 1 DBC's and $L_{x}$ for type 2 DBC's. This is in contrast to periodic boundary conditions, which violate duality but maintain equal coordination number for all lattice sites.

A third type of DBC has recently been suggested to us by $\mathrm{F}$. Y. Wu [34]; it can be defined as follows, and will be denoted as DBC type 3: consider an $L_{x} \times L_{y}$ lattice with $L_{x}=L+1, L_{y}=L$. Let all of the sites on the longer (horizontal) upper edge of the lattice be connected to a special adjoined point, via $L+1$ bonds, and similarly, let all of the sites on the lower edge of the lattice be connected by $L+1$ bonds to a second adjoined point. Finally, connect the two adjoined points by a single bond and let the sites on the vertical edges of the lattice have free boundary conditions (in the outward horizontal directions). This lattice has $N_{0}=N_{2}=L(L+1)+2$ and $N_{1}=2\left(L^{2}+L+1\right)=2\left(N_{0}-1\right)$ (so again, $q^{N_{0}-1-(1 / 2) N_{1}}=1$ in (14)). Evidently, type 3 DBC's share greater similarity with type 1 than type 2 since in types 1 and 3 no subsets of edge sites have periodic boundary conditions, while in type 2 the sites on the vertical edges do have PBC's. For a given value of $q$, the patterns of zeros which we have obtained with type 3 DBC's are indeed, similar to those with type 1.

In Figs. 1(a) and 1(b) we present calculations of complex-temperature zeros, in the complex $a$ plane, of the partition function for the $q=3$ Potts model on $8 \times 10$ lattices with duality preserving boundary conditions of types 1 and 2 , respectively. The positions of the singular points $a_{e}, a_{e}^{*}$ are marked with small circles on both of these plots. A comparison of these plots gives a quantitative measure of how the positions of the zeros can vary for different boundary conditions, and specifically for those which maintain duality. This extends previous published plots [30, 31], which showed that the pattern of zeros differs significantly when one uses duality-preserving, as opposed to duality-violating, boundary conditions. These comparisons demonstrated that once one specializes to duality-preserving BC's, the zeros in the $\operatorname{Re}(a)>0$ region lie nicely on the circle $|a-1|=\sqrt{q}$, whereas they lie close to, but not exactly on, this circle for duality-violating BC's. Among previously published calculations 

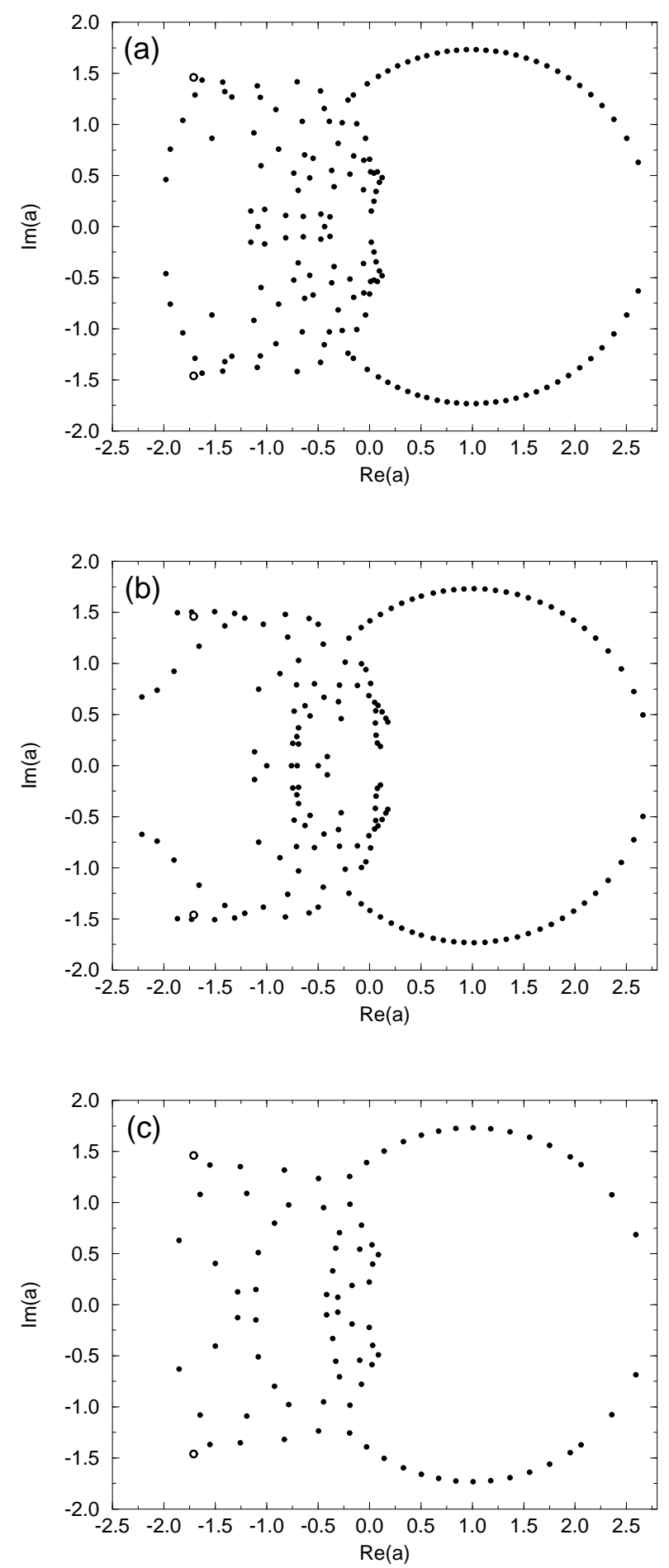

Figure 1: Plot of zeros of $Z$, in the $a$ plane, for the square-lattice $q=3$ Potts model on an $8 \times 10$ lattice with duality-preserving boundary conditions (DBC's) of (a) type 1; (b) type 2 ; and (c) on a $7 \times 6$ lattice with type 3 DBC's. The singularities $a_{e}, a_{e}^{*}$ are marked with $\circ$. 
with duality-preserving BC's, there is one, given as Fig. 11.1 in Ref. [30], on a lattice as large as the one that we use, and the pattern of zeros found there is very close to the pattern in our Fig. 1(b). In our figure, one can discern two outer-lying complex-conjugate arcs of zeros in the "northwest" and "southwest" quadrants, and the points $a_{e}, a_{e}^{*}$ lie near the ends of these arcs. In Fig. 1(a) there is more scatter among the zeros, but nevertheless, the points $a_{e}, a_{e}^{*}$ lie at the ends of subsets of zeros which can be associated with arcs. We have also performed analogous calculations of zeros on smaller lattices with similar results. For comparison, in Fig. 1(c) we show an exploratory calculation with type 3 DBC's. Taking into account that the lattice for Fig. 1(c) is somewhat smaller than that for Figs. 1(a,b), one sees that the distribution of zeros is similar to that in Fig. 1(a) with type 1 DBC's, especially in the region near $a_{e}, a_{e}^{*}$ and their dual images. From all of these calculations, we thus conclude that the observed patterns of zeros are consistent with the hypothesis that in the thermodynamic limit, the points $a_{e}, a_{e}^{*}$ are the ends of arcs contained in the complex-temperature boundary $\mathcal{B}$ which protrude into the FM phase. By duality, this is equivalent to the statement that the singularities at $\mathcal{D}\left(a_{e}\right), \mathcal{D}\left(a_{e}^{*}\right)$ are the endpoints of arcs contined in $\mathcal{B}$ which protrude into the PM phase.

\subsection{Singularities at $a=0$ and $a=-2$}

The exact solution (11) by Baxter shows that the point $a=0$, i.e. $T=0$ for $J<0$, is the antiferromagnetic critical point. By the duality property (14), it follows that the free energy is also singular, with the same singularity, at the dual to this point, namely, $a=-2 \equiv a_{s}$ given in eq. (12). From renormalization group methods, a mapping to a critical 6-vertex model, and studies of correlation functions, it has been concluded that at the AFM critical point is an essential singularity, with an essential zero in the free energy and an exponential divergence in the correlation length as $K \rightarrow-\infty$, i.e., $a \searrow 0$ [11]. Thus, if one assigns algebraic exponents $\alpha$ and $\nu$ for this AFM critical point, then $\alpha=-\infty, \nu=\infty$. By duality, the same singularity in the free energy occurs at the dual point $a=-2$.

We have addressed two questions concerning these singularities at $a=0$ and -2 : how do they connect with the complex-temperature phase boundary $\mathcal{B}$, (ii) how well do the low-temperature series detect the CT singular point at $a=-2$ ? For question (i), we first recall that the density $g$ of $\mathrm{CT}$ zeros along the curves comprising $\mathcal{B}$ in the vicinity of a generic singular point $a_{s}$ behaves as [13]

$$
g \sim\left|a-a_{s}\right|^{1-\alpha_{s}}
$$

where $a_{s}$ denotes a singular point and $\alpha_{s}\left(\alpha_{s}^{\prime}\right)$ denotes the corresponding specific heat exponent for the approach to $a_{s}$ from within the CTE PM (FM) phase. Since $\alpha=-\infty$ at $a=0$ 
and hence, by duality, $\alpha_{s}^{\prime}=-\infty$ at $a_{s}=-2$, there is a strong reduction in the density of CT zeros as one approaches these respective points $a=0$ and $a=-2$ along $\mathcal{B}$. This is consistent with what is observed with CT zeros calculated on finite lattices; one sees clear arcs of zeros and, e.g. for type 1 duality-preserving boundary conditions, these track toward the respective points $a=0$ and $a=-2$, ending some distance away from these points. (For type 2 DBC's, one also observes a slight curling tendency among the last few points on the curves.) From this tracking of the zeros toward $a=0$ (and hence, by duality, toward $a=-2$ ), we infer that the AFM critical point $a=0$ lies on a portion of $\mathcal{B}$ which connects with the continuation of the curves lying on (either part or all of) the unit circle $|a-1|=\sqrt{3}$ in such a way as to bound completely the complex-temperature extension of the PM phase. This is reminiscent of another model which has no AFM long-range order at any finite temperature, but an AFM critical point at $T=0$ [42, namely, the (isotropic, spin 1/2) Ising model on the triangular lattice. In that case, in terms of the variable $u=e^{-4 K}$, the complex-temperature phase boundary $\mathcal{B}$ consists of the union of the circle $|u+1 / 3|=2 / 3$ and the semi-infinite line segment running along the negative real $u$ axis from $-1 / 3$ to $-\infty$, or, equivalently, in the variable $u^{-1}$ analogous to $a$, it consists of the union of the circle $\left|u^{-1}-1\right|=2$ and the line segment $-3 \leq u^{-1} \leq 0$. The AFM critical point at $u^{-1}=0$ forms the right-hand end of this line segment and is connected to the rest of $\mathcal{B}$ by it. It is interesting to contrast this with situation in a model which is disordered and noncritical at $K=-\infty$, i.e. at $u^{-1}=0$. An example is provided by the (isotropic, spin 1/2) Ising model on the $3 \cdot 6 \cdot 3 \cdot 6$ (Kagomé) lattice [43, 44. Since the point $u^{-1}=0$ is noncritical, it must be true that this point is not connected to $\mathcal{B}$, and, indeed, one finds [18, 21] that is lies in the interior of the symmetric PM phase. The same behavior is found for the $3 \cdot 12^{2}$ lattice 21].

Returning to the $q=3$ Potts model, the property that the AFM critical point at $a=0$ is connected to the rest of $\mathcal{B}$ as described above implies, by duality, that the singular point at $a=-2$ lies on the dual image of the above-mentioned curve, which therefore encloses a complex-temperature phase and separates it completely from the (CTE of the) FM phase. Given the scatter of the zeros, it is not possible to make a very reliable inference for where these curves intersect the continuation of the circle $|a-1|=\sqrt{3}$ in the "northwest" and "southwest" quadrants of the complex a plane. We do remark that the zeros are consistent with the possibility that these intersection points are $a=e^{ \pm 2 \pi i / 3}$.

As regards the analysis of the low-temperature series, we find that these series are able to locate the singular point at $a_{s}=-2$, i.e., $z_{s}=-1 / 2$, but not very accurately. Representative results for diagonal dlog Padé approximants are given in Table 5, again based on the series calculated to $O\left(z^{47}\right)$ in Ref. [35. We do not show the results of the dlog Padé approximants $[N / D]$ with $N=D<13$ because these did not locate the $z=-1 / 2$ singularity with accept- 


\begin{tabular}{|c|c|c|}
\hline \hline$[N / D]$ & $z_{s}$ & $\gamma_{s}^{\prime}$ \\
\hline \hline$[14 / 14]$ & -0.478716 & 1.66 \\
\hline$[15 / 15]$ & -0.490833 & 2.31 \\
\hline$[16 / 16]$ & -0.487890 & 2.12 \\
\hline$[17 / 17]$ & -0.487889 & 2.12 \\
\hline$[18 / 18]$ & -0.487906 & 2.12 \\
\hline$[19 / 19]$ & -0.487844 & 2.11 \\
\hline$[20 / 20]$ & -0.487995 & 2.12 \\
\hline$[21 / 21]$ & -0.485409 & 1.99 \\
\hline \hline
\end{tabular}

Table 5: Values of $z_{s}$ and $\gamma_{s}^{\prime}$ from diagonal dlog Padé approximants to low-temperature series for $\bar{\chi}$ for $q=3$.

able accuracy (e.g., the [12/12] and [13/13] approximants gave -0.607830 and -0.572062 , respectively). As is evident from Table 5 , the series yield values of the susceptibility exponent $\gamma_{s}^{\prime} \sim 2$. Our previous work [19] has shown that there are subtleties in applying exponent relations like $\nu^{\prime} d=2-\alpha^{\prime}$ and $\gamma^{\prime}=\nu^{\prime}(2-\eta)$ at complex-temperature singularities. However, it is of interest to note that, since $\alpha_{s}^{\prime}=-\infty$, if the hyperscaling relation $\nu_{s}^{\prime} d=2-\alpha_{s}^{\prime}$ is valid, then $\nu_{s}^{\prime}=\infty$ and hence, assuming that the relation $\gamma_{s}^{\prime}=\nu_{s}^{\prime}\left(2-\eta_{s}\right)$ holds at $z_{s}$, it would follow that $\gamma_{s}^{\prime}=\infty$, i.e., $\chi$ would have an exponential divergence at this point. Of course, the series analysis cannot yield $\gamma_{s}^{\prime}=\infty$, but it does produce a rather large value. For reference, one may recall that in the case of the $2 \mathrm{D} \mathrm{O}(2)$ model, while the Kosterlitz-Thouless theory implies that $\chi$ diverges exponentially as $T \searrow T_{c}$, so that $\gamma=\infty$, the earlier series analyses gave a value of roughly $\gamma=3$ [45].

\subsection{Singularity at $a=1-3^{1 / 2}$}

The Baxter solution (10) shows that the free energy is also singular at the self-dual complextemperature point $a_{\ell}$ given in eq. (9) for $q=3$, viz., $a=1-\sqrt{3}$. There is considerable scatter of zeros in the vicinity of this point (less for Fig. 1(b) or Fig. 11.1 of Ref. 30] than in Fig. $1(\mathrm{~b}))$; however, the zeros are consistent with the inference that this singular point lies on a segment of the circle $|a-1|=\sqrt{3}$. As one approaches the regions near the intersection points discussed above, the scatter of zeros becomes too great to draw a firm conclusion about this part of $\mathcal{B}$. Nevertheless, we are able to infer that the point $a_{\ell}$ is completely separated from the FM phase by portions of the CT phase boundary $\mathcal{B}$. To see this, assume the contrary, 
i.e. that one can analytically continue from $z=0$ to $z_{\ell}=1 / a_{\ell}$. First, this would contradict the property that the singular point at $a=-2$ lies on a portion of $\mathcal{B}$ connecting it to the rest of $\mathcal{B}$ (the dual image of the curve connecting the physical AFM critical point to the rest of $\mathcal{B})$. Second, if $a_{\ell}$ were not completely separated from the FM phase, then one should be able to detect this singular point with the very long low-temperature series available. However, we found no evidence for a singularity at this point from our analysis of these series. The obvious conclusion is that $a_{\ell}$ lies in a region beyond the applicability of these series, i.e., beyond the border of the (CTE)FM phase.

\subsection{A Comment on $\operatorname{dim}(\mathcal{B})$ for $\operatorname{Re}(a)<0$}

We comment here on another feature of the pattern of zeros. For the $2 \mathrm{D}$ spin $1 / 2$ Ising model with isotropic spin-spin couplings, one knows from exact solutions that on most lattices, the zeros merge to form a 1-dimensional algebraic variety, i.e. the $\mathrm{CT}$ phase boundary $\mathcal{B}$. Even for isotropic couplings, there is a heteropolygonal lattice, i.e., the $4 \cdot 8^{2}$ lattice, for which this is not the case; the locus of points where the free energy is non-analytic forms a 2dimensional algebraic variety [21]. Moreover, for non-isotropic spin couplings, this is also true, even on the square lattice [46]. In both cases it is easy to see why this is true (see section 6 of Ref. [21]). The zeros of the 2D Ising model for higher spin values also appear to approach curves as the lattice size gets large [23]. For the 2D $q$-state Potts model, the zeros in the $R e(a)>0$ half-plane lie on a 1-dimensional curve, i.e., part of a circle [28]-32]. In the $\operatorname{Re}(a)<0$ region, we are not aware of any proof of this. However, we can observe that in the known cases with exact solutions, in the thermodynamic limit, the zeros either (i) form curves, or (ii) areas, but not both curves and areas. Thus, given that the zeros for $\operatorname{Re}(a)>0$ do form a curve, one would have a qualitatively new situation not previously encountered if some of the zeros in the $\operatorname{Re}(a)<0$ did form areas.

\section{$4 q=4$ Potts Model}

We have carried out exploratory analyses for higher- $q$ Potts models on the square lattice and have found evidence for singularities analogous to $z_{e}, z_{e}^{*}$ in each of the cases studied. We begin with the $q=4$ model. Like the $q=2,3$ cases, this model has a continuous, secondorder PM-FM transition. However, from the exact solution by Baxter on the manifold (11), it follows that the $q=4$ model does not have any AFM critical point. Note that the complex-temperature point $a=-1$ lies on the manifold (11).

We have analyzed the low-temperature series for the magnetization, susceptibility, and 
specific heat as before. These yield a consistent indication of singularities at the points listed in Table 4. (The values of $z_{e}$ from the $\bar{\chi}$ series exhibit somewhat greater scatter than those from the $m$ and $C$ series.) It is interesting that, to within the uncertainty, our numerical value for $\operatorname{Im}\left(a_{e}^{*}\right)$ can be fit by the exact expression $\operatorname{Im}\left(a_{e}^{*}\right)=\sqrt{3}$ for $q=4$. This and a similarly intriguing result which we find for $q=5$ suggest that there may be simple algebraic formulas for the singularities $a_{e}, a_{e}^{*}$. In view of the logarithmic confluent singularities which are known to occur at the physical PM-FM transition in this model [47], it would be useful in future work to carry out a more sophisticated analysis of the series including such confluent singularities also at the complex-temperature singularities; however, in the present exploratory work we have not done this. From our study of the low-temperature series for $m$, we find the exponent $\beta_{e}=-0.12(1)$, so that, as before, the magnetization diverges at $z_{e}, z_{e}^{*}$. This rigorously implies [24] that $\chi$ also diverges at these points.

In order to see how these singularities connect to the complex-temperature phase boundary $\mathcal{B}$ for the model, we have carried out a calculation of $\mathrm{CT}$ zeros of the partition function on various lattices with duality-preserving boundary conditions. In Figs. 2(a) and 2(b) we show calculations of these zeros, in the complex $a$ plane, for a $6 \times 6$ lattice with type 1 and type 2 DBC's, respectively. Previously published calculations of zeros for this model include plots for strips $\left(L_{x} \times 32\right.$ for $L_{x}=4,6$, and 8 ; and $\left.10 \times 16\right)$ [29] and a plot for a $4 \times 4$ lattice with type 2 DBC's. We use symmetric lattices since in taking the thermodynamic limit on an $L_{x} \times L_{y}$ lattice, if $L_{x} / L_{y}$ deviates strongly from unity, the results can involve 1-dimensional artifacts. For type 1 DBC's (Fig. 2(a)) one sees a clear indication of a complex-conjugate pair of arcs of zeros in the "northwest" and "southwest" quadrants, with the singularities at $a_{e}, a_{e}^{*}$ forming the endpoints of these arcs. These arcs are not as clear with type 2 DBC's ((Fig. 2(b)), but again, the points $a_{e}, a_{e}^{*}$ lie at the ends of subsets of zeros which can be associated with arcs. A calculation with type 3 DBC's is included as Fig. 2(c). The resultant pattern is very similar to that in Fig. 2(a) with type 1 DBC's, to an even greater extent than in the $q=3$ model. The patterns of zeros in Figs. 2(a)-2(c) are all in good agreement with the above-mentioned property that the model is not critical even at $a=0$, i.e., that it has no AFM critical point even at $T=0$. For the plot in Fig. 2(b) with type 2 DBC's, the zeros are consistent with the expectation from the Baxter solution (11) that the complex-temperature phase boundary $\mathcal{B}$ passes through the point $a=-1$ (equivalently for this $q=4$ case, $x=-1$ ). In contrast, for the plots in Figs. 2(a) and 2(c), with type 1 and type 3 DBC's, respectively, there are no zeros near to, or easily extrapolated toward, $a=-1$. A clarification of the situation in the vicinity of this point merits further study on larger lattices. 

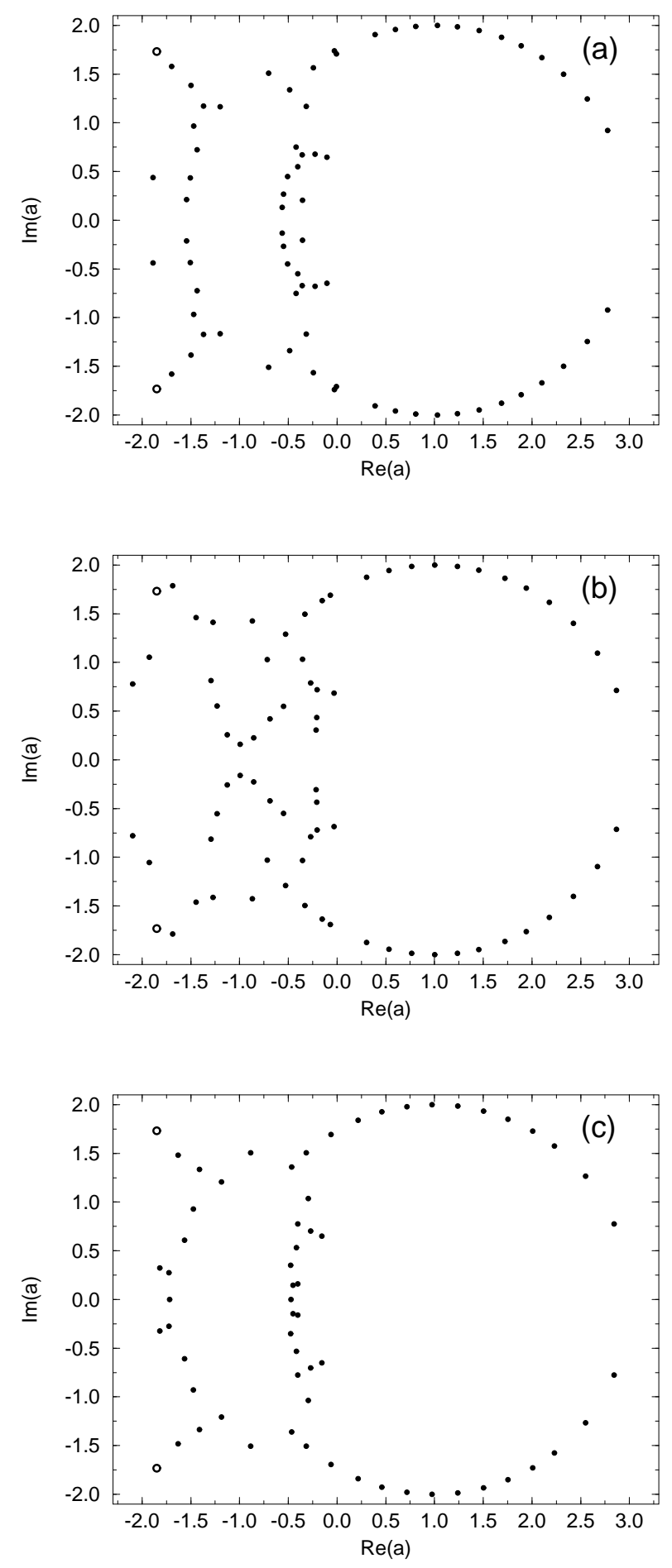

Figure 2: Plot of zeros of $Z$, in the $a$ plane, for the square-lattice $q=4$ Potts model on a $6 \times 6$ lattice with DBC of (a) type 1; (b) type 2; and (c) on a $6 \times 5$ lattice with DBC's of type 3. Notation is as in Fig. 1. 


\section{Higher- $q$ Potts Model}

We proceed to square-lattice Potts models with $q \geq 5$, for which the physical PM-FM phase transition is first-order. Of course, a series analysis does not, in general, yield an accurate determination of the location of the phase transition point for a first-order transition. However, there is no obvious reason why this should be a drawback for our study of the complex-temperature arc endpoint singularities $z_{e}, z_{e}^{*}$, since these yield a strong signal in the form of a divergent magnetization. In Table 6 we list the results for $z_{e}$ and corresponding exponent $\beta_{e}$ from diagonal dlog Padé approximants to the low-temperature series for $m$. We obtain similar results for $z_{e}$ from the susceptibility and specific heat series. From these diagonal (and near-diagonal) dlog Padé approximants, we we obtain the value for $z_{e}$ listed in Table 4. This is an intriguing result, since, to within the uncertainty, our determination is consistent with the following exact analytic formula:

$$
a_{e}, a_{e}^{*}=2(-1 \mp i) \quad \text { for } q=5
$$

Of course, in the absence of an exact solution of the model, we cannot exclude the possibility that this agreement is fortuitous, but it motivates one to think further about simple analytic expressions for the location of the arc endpoints which we have discovered. From our series analysis, we find that the magnetization diverges at these points with exponent $\beta_{e}=-0.11(1)$. As noted above, this implies [24] that $\bar{\chi}$ must also diverge at these points, and our analysis of the low-temperature series for $\bar{\chi}$ yields the exponent $\gamma_{e}^{\prime}=1.2(1)$.

We find the same generic features for all of the $q$ values that we have analyzed, viz., complex-conjugate singularities at points $z_{e}, z_{e}^{*}$. These thus appear to be a general feature of the $q$-state Potts model on the square lattice for $q \geq 3$. Besides the case $q=5$, we have made exploratory studies of the low-temperature expansions for the cases $q=6,7$, and 8 . We find $z_{e}, z_{e}^{*}=-0.23 \pm 0.24 i,-0.21 \pm 23 i$, and $z_{e}=-0.20 \pm 22 i$ for $q=6,7$, and 8 , respectively. Thus, the magnitude $\left|z_{e}\right|\left(\left|a_{e}\right|\right)$ decreases (increases) as $q$ increases. Note that if, in the $x$ plane, the complex-conjugate arcs retract toward their respective points of origin and finally disappear in the $q \rightarrow \infty$ limit, as is required in order for the complex-temperature phase boundary $\mathcal{B}$ to reduce to the unit circle $|x|=1$ in this limit, it is necessary that for large $q$, $\left|R e\left(a_{e}\right)\right|$ and $\left|\operatorname{Im}\left(a_{e}\right)\right|$ grow like $\sqrt{q}$. We find that $\beta_{e}$ increases from $\sim-0.11$ for $q=6$ to $\sim-0.10$ for $q=8$.

In Figs. 3 and 4 we show our calculation of CT zeros of $Z$ for $q=5$ and $q=6$ on a $6 \times 6$ lattice with type 2 DBC's. (We have checked that other DBC's give results which also support our conclusions.) Although there is considerable scatter of zeros in the $\operatorname{Re}(a)<0$ region, the pattern is again consistent with the conclusion that the singularities at $a_{e}, a_{e}^{*}$ lie 


\begin{tabular}{|l|l|l|}
\hline \hline$[N / D]$ & $z_{e}$ & $\beta_{e}$ \\
\hline$[10 / 10]$ & $-0.248723+0.250655 i$ & -0.1023 \\
\hline$[11 / 10]$ & $-0.248197+0.250780 i$ & -0.1004 \\
\hline$[10 / 11]$ & $-0.248617+0.250694 i$ & -0.1019 \\
\hline$[11 / 11]$ & $-0.249536+0.249950 i$ & -0.1034 \\
\hline$[12 / 11]$ & $-0.251131+0.250021 i$ & -0.1105 \\
\hline$[11 / 12]$ & $-0.251182+0.250694 i$ & -0.1142 \\
\hline$[12 / 12]$ & $-0.251032+0.250976 i$ & -0.1146 \\
\hline$[13 / 12]$ & $-0.250982+0.250979 i$ & -0.1143 \\
\hline$[12 / 13]$ & $-0.250978+0.250974 i$ & -0.1142 \\
\hline$[13 / 13]$ & $-0.251028+0.250979 i$ & -0.1146 \\
\hline$[14 / 13]$ & $-0.251528+0.251159 i$ & -0.1190 \\
\hline$[13 / 14]$ & $-0.251281+0.250904 i$ & -0.1157 \\
\hline$[14 / 14]$ & $-0.251527+0.251148 i$ & -0.1189 \\
\hline$[15 / 14]$ & $-0.251527+0.251158 i$ & -0.1190 \\
\hline$[14 / 15]$ & $-0.251475+0.251197 i$ & -0.1188 \\
\hline$[15 / 15]$ & $-0.251524+0.251171 i$ & -0.1190 \\
\hline$[16 / 15]$ & $-0.251529+0.251161 i$ & -0.1190 \\
\hline$[15 / 16]$ & $-0.251667+0.251138 i$ & -0.1199 \\
\hline$[16 / 16]$ & $-0.251668+0.251140 i$ & -0.1199 \\
\hline$[17 / 16]$ & $-0.251631+0.251079 i$ & -0.1191 \\
\hline$[16 / 17]$ & $-0.251667+0.251138 i$ & -0.1199 \\
\hline$[17 / 17]$ & $-0.251710+0.251167 i$ & -0.1205 \\
\hline$[18 / 17]$ & $-0.251396+0.251142 i$ & -0.1181 \\
\hline$[17 / 18]$ & $-0.251644+0.251144 i$ & -0.1198 \\
\hline$[18 / 18]$ & $-0.252009+0.251663 i$ & -0.1282 \\
\hline$[19 / 18]$ & $-0.251915+0.251720 i$ & -0.1267 \\
\hline$[18 / 19]$ & $-0.251910+0.251717 i$ & -0.1266 \\
\hline$[19 / 19]$ & $-0.251929+0.251724 i$ & -0.1270 \\
\hline \hline & & \\
\hline
\end{tabular}

Table 6: Values of $z_{e}$ and $\beta_{e}$ from dlog Padé approximants to low-temperature series for $m$, for $q=5$. 


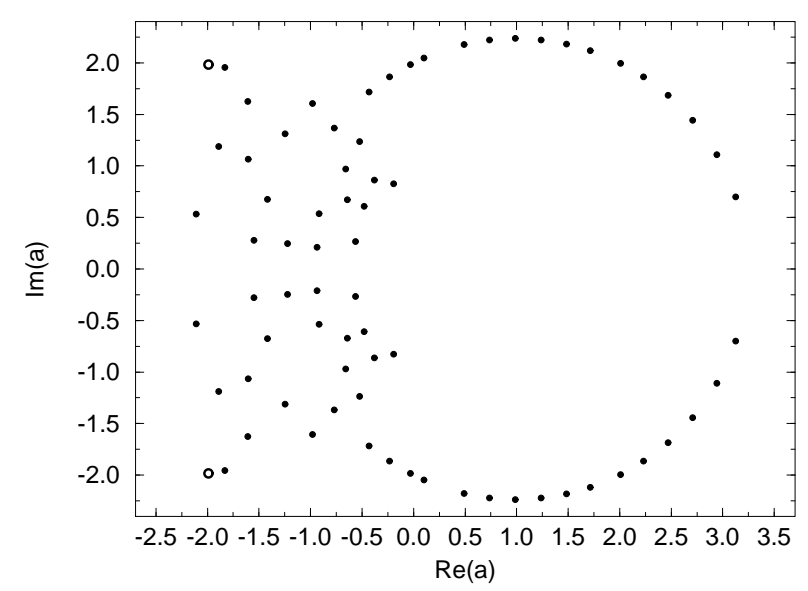

Figure 3: Plot of zeros of $Z$, in the $a$ plane, for the square-lattice $q=5$ Potts model on a $6 \times 6$ lattice with DBC of type 2 . Notation is as in Fig. 1 .

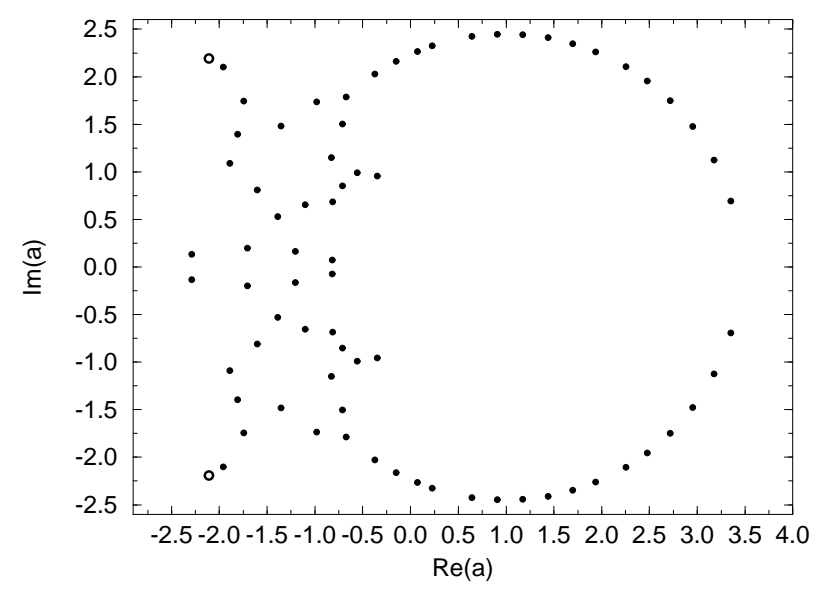

Figure 4: Plot of zeros of $Z$, in the $a$ plane, for the square-lattice $q=6$ Potts model on a $6 \times 6$ lattice with DBC of type 2 . Notation is as in Fig. 1 . 
at the end of arcs of zeros, so that in the thermodynamic limit these points are endpoints of arcs of singularities which connect with the rest of the complex-temperature phase boundary $\mathcal{B}$.

\section{Conclusions}

In summary, we have used analyses of low-temperature series expansions to study complextemperature singularities in the square-lattice $q$-state Potts model. We have found singularities at complex-conjugate pairs of points and, by means of comparison with patterns of partition function zeros, have obtained support for the inference that in the thermodynamic limit these are endpoints of arcs lying on the complex-temperature phase boundary $\mathcal{B}$. At these points, the magnetization diverges, in agreement with an earlier conjecture which we had formulated. This guarantees that the susceptibility also diverges at these points, and our series analyses are in accord with this. Our work includes several intriguing findings, including the likely exponent value $\beta_{e}=-1 / 8$ for $q=3$ and an inference of an exact formula (26) for the endpoint singularities for $q=5$. From the duality of the model, it follows that these arcs protruding into the complex-temperature extension of the FM phase are accompanied by their dual images, i.e. arcs protruding into the CT extension of the PM phase. Our new results further elucidate the complex-temperature phase diagrams of square-lattice Potts models.

We thank Prof. F. Y. Wu for the helpful private communication [34]. This research was supported in part by the NSF grant PHY-93-09888.

\section{References}

[1] R. B. Potts, Proc. Camb. Phil. Soc. 48, 106 (1952).

[2] C. Domb, J. Phys. A 7, 1335 (1974).

[3] S. Alexander, Phys. Lett. A54, 353 (1975); A. N. Berker, S. Oslund, and F. Putnam, Phys. Rev. B17, 3650 (1978); E. Domany, M. Schick, J. S. Walker, and R. B. Griffiths, Phys. Rev. B18, 2209 (1978).

[4] R. J. Baxter, J. Phys. C 6, L445 (1973); R. J. Baxter, H. N. V. Temperley, and S. Ashley, Proc. Roy. Soc. London, Ser. A 358, 535 (1978); R. J. Baxter, J. Stat. Phys. 28, 1 (1982). 
[5] R. J. Baxter, Proc. Roy. Soc. London, Ser. A 383, 43 (1982).

[6] M. P. M. den Nijs, J. Phys. A 12, 1857 (1979); Phys. Rev. B27, 1674; J. L. Black and V. J. Emery, Phys. Rev. B23, 429 (1981); B. Nienhuis, J. Appl. Phys. 15, 199 (1982).

[7] A. A. Belavin, A. M. Polyakov, and A. B. Zamolodchikov, Nucl. Phys. B241, 333 (1984); D. Friedan, Z. Qiu, and S. Shenker, Phys. Rev. Lett. 52, 1575 (1984); V. S. Dotsenko, Nucl. Phys. B235, 54 (1984).

[8] F. Y. Wu, Rev. Mod. Phys. 54, 235 (1982).

[9] C. N. Yang and T. D. Lee, Phys. Rev. 87 (1952) 404; T. D. Lee and C. N. Yang, ibid. 87 (1952) 410.

[10] M. E. Fisher, Lectures in Theoretical Physics (Univ. of Colorado Press, 1965), vol. 7C, p. 1.

[11] S. Katsura, Prog. Theor. Phys. 38, 1415 (1967); Y. Abe and S. Katsura, ibid. 43, 1402 (1970)

[12] S. Ono, Y. Karaki, M. Suzuki, and C. Kawabata, J. Phys. Soc. Jpn. 25, 54 (1968).

[13] R. Abe, Prog. Theor. Phys. 38, 322 (1967).

[14] C. J. Thompson, A. J. Guttmann, B. W. Ninham, J. Phys. C 2, 1889 (1969); A. J. Guttmann, ibid, 1900 (1969); C. Domb and A. J. Guttmann, J. Phys. C 3, 1652 (1970).

[15] A. J. Guttmann, J. Phys. A 81236 (1975).

[16] R. B. Pearson, Phys. Rev. B26, 6285 (1982); C. Itzykson, R. B. Pearson, and J. B. Zuber, Nucl. Phys. B220, 415 (1983).

[17] G. Marchesini and R. Shrock, Nucl. Phys. B318, 541 (1989).

[18] R. Abe, T. Dotera, and T. Ogawa, Prog. Theor. Phys. 85, 509 (1991).

[19] V. Matveev and R. Shrock, J. Phys. A 28, 1557 (1995).

[20] V. Matveev and R. Shrock, J. Phys. A 28, 4859 (1995).

[21] V. Matveev and R. Shrock, J. Phys. A 28, 5235 (1995).

[22] V. Matveev and R. Shrock, Phys. Rev. E53, 254 (1996). 
[23] V. Matveev and R. Shrock, J. Phys. A (Lett.) 28 L533 (1995); Phys. Lett. A204 353 (1995).

[24] V. Matveev and R. Shrock, J. Phys. A 29, 803 (1996).

[25] I. G. Enting, A. J. Guttmann, and I. Jensen, J. Phys. A 27, 6963 (1994).

[26] I. Jensen, A. J. Guttmann, and I. G. Enting, Melbourne preprint cond-mat/9604080.

[27] J. M. Maillard and R. Rammal, J. Phys. A 16, 353 (1983).

[28] P. P. Martin and J. M. Maillard, J. Phys. A 19, L547 (1986).

[29] P. P. Martin, J. Phys. A 19, 3267 (1986). See also P. P. Martin, ibid., 20, L601 (1986).

[30] P. P. Martin, Potts Models and Related Problems in Statistical Mechanics (World Scientific, Singapore, 1991).

[31] C. N. Chen, C. K. Hu, and F. Y. Wu, Phys. Rev. Lett. 76, 169 (1996).

[32] F. Y. Wu, G. Rollet, H. Y. Huang, J. M. Maillard, C. K. Hu, and C. N. Chen, Phys. Rev. Lett. 76, 173 (1996).

[33] The $q=\infty$ limit of the Potts model is solvable; see P. A. Pearce and R. B. Griffiths, J. Phys. A 13, 2143 (1980). For some expansions in $1 / \sqrt{q}$, see T. Bhattacharya, R. Lacaze, and A. Morel, hep-lat/9601012 and references therein.

[34] Our type 2 duality preserving boundary conditions (DBC's) turn out to be the same as one of the DBC's alluded to in the reference to unpublished calculations of zeros with other DBC's (the other being type 3) in Ref. [31]. We thank Prof. F. Y. Wu for kindly informing us of this and suggesting that we try his type 3 DBC's.

[35] K. M. Briggs, I. G. Enting, and A. J. Guttmann, J. Phys. A 27, 1503 (1994).

[36] T. Kihara, Y. Midzuno, T. Shizume, J. Phys. Soc. Jpn. 9, 681 (1954); J. Straley and M. E. Fisher, J. Phys. A 6, 1310 (1973); J. Adler, I. G. Enting, and V. Privman, ibid. 16, 1967 (1982). See Ref. [35] for further references.

[37] G. Bhanot, M. Creutz, U. Glässner, I. Horvath, J. Lacki, K. Schilling, and J. Weckel, Phys. Rev. B48, 6183 (1993). 
[38] The results obtained in Ref. [5] involved a mapping from the Potts model to a six-vertex model. A complication pertaining to a mismatch between the boundary conditions for the Potts and vertex model was noted there. See also H. Saleur, Commun. Math. Phys. 132, 657 (1990).

[39] M. P. N. den Nijs, Physica A 95, 449 (1979); J. Phys. A 12, 1857 (1979) and references therein.

[40] A. J. Guttmann, in Phase Transitions and Critical Phenomena, Domb, C. and Lebowitz, J., eds. (Academic Press, New York, 1989)) vol. 13.

[41] M. P. Nightingale and M. Schick, J. Phys. A, 15, L39 (1982); M. P. M. den Nijs, M. P. Nightingale, and M. Schick, Phys. Rev. B26, 2490 (1982); J.-S. Wang, R. H. Swendsen, and R. Kotecký, Phys. Rev. B42, 2465 (1990); S. J. Ferreira and A. D. Sokal, Phys. Rev. B51, 6727 (1995).

[42] The criticality at $K=-\infty$ is evident, e.g., from the fact that $\left\langle\sigma_{0} \sigma_{r}\right\rangle$ decays like a power, $r^{-1 / 2}$ (multiplied by an oscillatory trigonometric factor), rather than exponentially; see J. Stephenson, J. Math. Phys. 5, 1009 (1964), 11, 420 (1970).

[43] An Archimedean heteropolygonal lattice is defined by the symbol $p_{1} \ldots p_{n}$, meaning that as one makes a circuit around a site on the lattice, one traverses the polygons $p_{1}, p_{2}, \ldots$ $p_{n}$; see Ref. 21].

[44] K. Kano and S. Naya, Prog. Theor. Phys. 10, 158 (1953); A. Sütö, Z. f. Physik B44, 121 (1981).

[45] H. E. Stanley and T. Kaplan, Phys. Rev. Lett. 17, 913 (1966); M. A. Moore, ibid., 23, 861 (1969).

[46] W. van Saarloos and D. Kurtze, J. Phys. A 17, 1301 (1984); J. Stephenson and R. Couzens, Physica 129A, 201 (1984); D. Wood, J. Phys. A 18, L481 (1985); J. Stephenson, Physica 136A, 147 (1986); J. Stephenson and J. van Aalst, ibid. 136A, 160 (1986).

[47] M. Nauenberg and D. J. Scalapino, Phys. Rev. Lett. 44, 837 (1980); J. Cardy, M. Nauenberg and D. J. Scalapino, Phys. Rev. B22, 2560 (1981). 